\title{
COVID-19:
}

\section{Comparison of the Response in Rwanda, South Africa and Zimbabwe}

\author{
Tafadzwa Dzinamarira MPH PhD, Munyaradzi P. Mapingure MD MPH, Gallican N. Rwibasira MD MPH, \\ Solomon Mukwenha MPH, Godfrey Musuka DVM MPhil MSc (Med)
}

\begin{abstract}
The COVID-19 pandemic has had an impact worldwide with regions experiencing varying degrees of severity. African countries have mounted different response strategies eliciting varied outcomes. Here, we compare these response strategies in Rwanda, South Africa and Zimbabwe and discuss lessons that could be shared. In particular, Rwanda has a robust and coordinated national health system that has effectively contained the epidemic. South Africa has considerable testing capacity, which has been used productively in a national response largely funded by local resources but affected negatively by corruption. Zimbabwe has an effective point-of-entry approach that utilizes an innovative strategic information system. All three countries would benefit having routine meetings to share experiences and lessons learned during the COVD-19 pandemic.
\end{abstract}

KEYWORDS COVID-19, SARS-CoV-2, epidemics, pandemic, Africa, Zimbabwe, Rwanda, South Africa

\section{INTRODUCTION}

COVID-19 emerged in China in late 2019 and has spread around the globe, infecting nearly 200 million and leading to more than 3 million deaths. To date, SARS-CoV-2, the virus that causes COVID-19, has spread to almost every region of the world.[1,2] Affected countries have mounted different response strategies with the overall goal of minimizing morbidity and mortality and associated socioeconomic impacts.[3] Drawing experience from the 2014 Ebola virus disease crisis in West Africa, African leaders are keenly aware that failure to contain COVID-19 would threaten health, prosperity and security.[4]

In this manuscript, we compare COVID-19 response strategies in Rwanda, South Africa and Zimbabwe. We specifically focused on these three countries as the authors are involved in the COVID-19 response in these countries and therefore would have insights sufficient for detailed comparisons. All figures in this study, including those in Table 1, correspond to February 25, 2021, when this paper was drafted. The Rwandan COVID-19 pandemic has had over 18,500 positive cases and more than 250 deaths. South Africa has had the worst COVID-19 outbreak among the

IMPORTANCE Comparing COVID-19 responses between countries is useful as countries are learning in real time from practicing in adaptive systems; a necessity given the nature of the pandemic. This paper compares the diversity of responses to the pandemic in different African countries. This contributes to ongoing efforts to understand and adapt to the demands of the COVID-19 pandemic through shared experiences. three countries with 1.5 million confirmed cases and over 49,600 deaths. As of the same date, Zimbabwe had recorded over 35,900 confirmed-positive cases with over 1450 deaths.[5]

\section{DEVELOPMENT}

For this study, we conducted a literature review of COVID-19 response strategies across the three countries. We searched for articles published in English on: WHO's website; peer-reviewed articles on Google Scholar and PubMed; official public health websites operated by the respective governments of each country; and newspaper articles written and published within each country. We used the following keywords: COVID-19; response; Africa; Rwanda; South Africa; Zimbabwe; and other subject specific terms such as surveillance; infection prevention and control; policy. We used the Boolean operators AND and OR to separate the keywords. For instance, the search strategy used in PubMed was ("COVID19"[All Fields] OR "COVID-19"[MeSH Terms] OR “COVID-19 Nucleic Acid Testing"[All Fields] OR "covid-19 nucleic acid testing"[MeSH Terms] OR "COVID-19 Serological Testing"[All Fields] OR "covid-19 serological testing"[MeSH Terms] OR "COVID-19 Testing"[All Fields] OR "covid-19 testing"[MeSH Terms] OR "Severe Acute Respiratory Syndrome Coronavirus 2"[All Fields] OR "coronavirus"[All Fields] OR response[All Fields] AND ("africa"[MeSH Terms] OR "africa"[All Fields]) OR ("rwanda"[MeSH Terms] OR "rwanda"[All Fields]) OR ("south africa"[MeSH Terms] OR ("south"[All Fields] AND "africa"[All Fields]) OR "south africa"[All Fields]) OR ("zimbabwe"[MeSH Terms] OR "zimbabwe"[All Fields]) OR ("epidemiology"[Subheading] OR "epidemiology"[All Fields] OR "surveillance"[All Fields] OR "epidemiology"[MeSH Terms] OR "surveillance"[All Fields]) AND ("infections"[MeSH Terms] OR "infections"[All Fields] OR "infection"[All Fields]) AND ("prevention and control"[Subheading] OR ("prevention"[All Fields] AND "control"[All Fields]) OR "prevention and control"[All Fields]) AND ("policy"[MeSH Terms] OR "policy"[All Fields]) Consistent with standard literature review methodology, some steps, such appraising evidence quality (a standard step in systematic reviews) were omitted.

To allow for a well-rounded comparison, the information gathered was structured and is presented according to ten pre-established comparison domains:

- Coordination, planning and monitoring

- Policy framework

- Risk communication and community engagement

- Surveillance, rapid response teams and case investigation

- Infection prevention and control

- Case management and continuity of essential services

- National laboratory system

- Role of private sector in the national response

- Points of entry

- COVID-19 logistics, supply and procurement implementation/ operational plan.

These comparison domains were adopted from the monitoring and evaluation framework for the COVID-19 response in WHO's 
African Region.[6] Using this framework, we discuss key findings in each country's response based on the ten established domains of comparison. We also present key background information and COVID-19 related statistics for each country to provide context (Table 1).

Coordination, planning and monitoring Governments of these countries have made COVID-19 responses a national priority with each one instituting a variety of measures aimed at curbing the virus's spread. The Rwandan government quickly formed a Joint Task Force to plan, coordinate and monitor the response to the COVID-19 epidemic.[10] In fact, the Task Force was formed before the country recorded its first confirmed case. This organization was comprised of various stakeholders in the Ministry of Health $(\mathrm{MOH})$ and chaired by the Prime Minister. In South Africa, literature revealed evidence of coordinating bodies. An inter-ministerial organization comprised of the Ministry of Health, Department of Defense, Ministry of Public Works, Ministry of Justice and Correctional Services, and Ministry of Basic and Higher Education, among other line ministries, was set up to coordinate COVID-19 response with assistance from an advisory board composed of medical experts.[11] As is the case with Rwanda, this organization was formed before any known cases were reported in the country.

Similarly, Zimbabwe prepared a National Preparedness and Response Plan tailored to minimizing COVID-19 morbidity and mortality and mitigating the pandemic's socioeconomic consequences.[12] It included prevention, containment and mitigation strategies for different COVID-19 transmission scenarios. Given the vast number of health-related (and nonhealth-related) actors and stakeholders who would be potentially involved in addressing the outbreak, it was deemed important that they all work under one framework with clearly articulated roles and responsibilities. This was designed to ensure efficient allocation of scarce resources as well as alignment by all stakeholders in the overall strategic direction of the response. Two levels of coordination were set up to ensure a robust pandemic response:

Table 1: Key statistics on Rwanda, South Africa and Zimbabwe

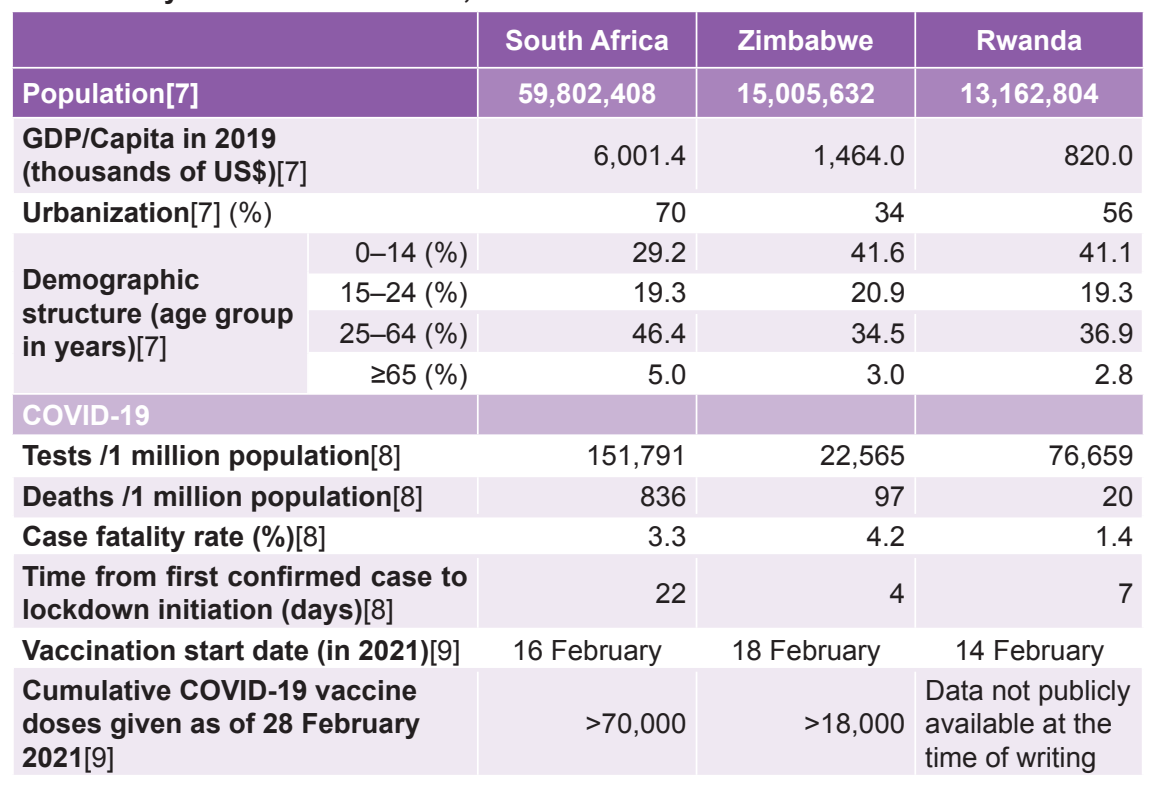

Unless otherwise stated, figures correspond to information from sources obtained as of February 25, 2021. first is the Inter-Ministerial Task Force (chaired by one of the vice presidents and responsible for overall response coordination). The national public health emergency management mechanisms work with all relevant ministries including education, travel and tourism, public works, environment, social protection, agriculture, trade, and industry and finance to provide coordinated management of COVID-19 preparedness and response. Implementation of the plan was rolled out to provinces throughout the country. The second level Health Sector Coordination is for activities both within the Ministry of Health itself and within the broader health sector (which includes local government, the private sector, nonprofits and other related stakeholders).

For this domain, all three countries demonstrated strong government involvement and willingness to set up institutions to lead the response. South Africa and Zimbabwe have involved more line ministries in the national coordination taskforce to improve inter-ministerial cooperation and streamline the delegation of responsibilities.

Policy framework Within a few weeks of its formation, the Rwandan Joint Task Force for COVID-19 put forward a nationa policy on COVID-19 prevention within communities, places prone to large gatherings of people, markets, and other crowded places such as bus stations.[13] Clinical tools and guidelines were quickly developed and shared to help heath care providers manage testing, and also offer instructions as to how to access services after exposure.[13] By March 2020, various containment and mitigation measures had been put in place.[14] These included lockdowns, restricted movement between Kigali and other provinces, reducing the number of traders in markets and closing markets with high rates of transmission, closing schools and churches, and isolating regions with transmission clusters in lockdown.

A similar response was observed in South Africa. Building on the declaration of COVID-19 as a national disaster by the Minister of Cooperative Governance and Traditional Affairs, a national policy including various clinical tools and guidelines were put in place in South Africa.[15]

At the time this article was written, our search could not find a COVID-19 policy on the Zimbabwean Ministry of Health and Child Care website. However, the Ministry of Health and Child Care had published a COVID 19 National Preparedness and Response Plan.[3] The plan includes prevention, containment and mitigation strategies for different COVID-19 transmission scenarios.[16]

The existence of a COVID-19 national policy that covers the major response areas in public domain or on the government websites is critical for guiding national responses. In this context, Rwanda moved with respective alacrity, establishing policies that allowed for rapid introduction of strict prevention and control interventions.

Risk communication and community engagement Our literature search showed that all 
three countries have employed the use of SMS text alerts, videos, infographics and posters to alert the public to the dangers of COVID-19. Public and private radio and television were also used to disseminate information. A compendium of key messages has been developed and these guide other partners involved in the COVID-19 response in the development of information, education and communication activities. There were no clear differences among the three countries in risk communication and community engagement strategies.

Surveillance, rapid response teams and case investigation All three countries have set up systems actively involved in case detection, quarantine and isolation. In South Africa, community health workers conduct house-to-house screening and testing, especially in vulnerable communities. In Rwanda and Zimbabwe, rapid response teams investigate suspected cases and support the surveillance task force at subnational levels through data reporting, capacity building and supportive visits.[3] However, in Zimbabwe, there were reports of rapid response teams in the first wave (July-August 2020),[17] but at the time of this writing there was no literature available which would reveal whether the number of these increased during Zimbabwe's COVID-19 resurgence from mid-December 2020 through the end of January 2021.

Infection prevention and control A major component of the COVID-19 response has been infection prevention and control (IPC). All three countries have implemented COVID-19 IPC response plans, albeit with logistical and personnel challenges. [17] In Rwanda and South Africa, various mitigation measures were put in place; including limiting visits to healthcare facilities, screening all patients for COVID-19 symptoms and patient triage. Healthcare facility staff receive routine training on COVID-19 risk reduction.[18] Similarly, in Zimbabwe, IPC reference materials for reducing COVID-19 exposure risks were developed rapidly and distributed in health facilities and within communities in an effort to better capacitate healthcare workers (HCWs) to provide safe community environments. The literature in this review revealed that the Zimbabwean IPC response was affected by staffing shortages, lack of motivation among HCWs and personal protective equipment (PPE) shortages.[17] In this regard, Rwanda and South Africa had more engaged volunteer health workers[19] and reassigned HCWs who had switched to employment outside the health sector.

Case management and continuity of essential services All three countries have active case management systems functioning at varying degrees of efficiency. Their objectives are to provide timely high-quality care for COVID-19 patients; to ensure adequate capacity for managing COVID-19 cases during all phases of the pandemic, including during case surges; and to ensure routine essential health service delivery continuity and utilization during all phases of the pandemic and beyond.

In Rwanda, clinical management guidelines were continuously updated to reflect new guidance from WHO. South Africa utilized various action plans recommended by $\mathrm{WHO}$, among them mapping vulnerable populations, as well as public and private health facilities and workforces, thereby identifying alternative facilities for treatment. Similarly, Zimbabwe released guidelines for the clinical management of COVID-19 that covered testing, case management, antiviral treatment and patient discharge. While there was evidence on the existence of such guidelines in all three countries, our literature search did not reveal evidence on the quality or efficiency of case management.

Essential services in Rwanda remained functional, if depleted. However, movement restrictions and bans on public transportation impeded access to non-COVID healthcare services. For instance, one study reported that less than half of HIV-positive patients attended their antiretroviral collection clinic appointments during the first lockdown period in March-April 2020.[20] In South Africa, the healthcare facilities were continuously assessed to ensure continued capacity in delivering primary and other essential services. Furthermore, the private for-profit healthcare system in South Africa in general is very active in care management; mainly attending to patients with medical insurance.

In Zimbabwe, although essential services remained open during the lockdown period, movement restrictions and fear of contracting COVID-19 at healthcare facilities affected utilization of such services for other public health threats.[21] A report by the Zimbabwean Ministry of Health and Child Care revealed that during the period of April-June 2020 there was a 59\% reduction in the number of clients tested for HIV who received their results; $15 \%$ reduction in the distribution of HIV self-test kits; $99 \%$ reduction in voluntary medical male circumcisions performed; a $49 \%$ reduction in patients tested for syphilis; $46 \%$ reduction in pregnant women booking for first prenatal appointment; $51 \%$ reduction in newly diagnosed HIV patients initiated on antiretroviral therapy and a $29 \%$ decline in viral load sample collection in Zimbabwe. One observation is that Rwanda and Zimbabwe could benefit from scaling up telehealth utilization, as reportedly used in South Africa,[22] to support essential services' continuation during the pandemic.

National laboratory system COVID-19 tests in Rwanda were initially performed at the National Reference Laboratory (NRL) before a new testing strategy was introduced to decentralize capacity through peripheral district laboratories. The Rwandan laboratory system started off (in March 2020) with the capacity to test close to 1000 samples per day but in 4 months (by July 2020), the testing capacity increased 15-fold with a shift from manual RNA extraction to an automated system providing results more quickly (pooling system).[11,23,24] This was due in part to the introduction of a mobile laboratory unit in May 2020 that doubled the country's COVID-19 testing capacity, its mobility facilitating mass testing throughout the country.

The South African COVID-19 response laboratory is led by the National Institute of Communicable Diseases (NICD). Rapid testing expansion was enabled by a large network of private and National Health Laboratory Services (NHLS) laboratories. As with Rwanda, South Africa has employed mobile laboratories to expand testing.

Zimbabwe has a national-level laboratory system whose objectives include capacitating laboratories to perform molecular diagnosis using real-time reverse transcription polymerase chain reaction (RT-PCR) with demonstrated quality and biosafety; ensuring adequate supplies of test kits and reagents; increasing access to testing at provincial level using GeneXpert (Cepheid); strengthening COVID-19 testing support systems including data collection and analysis, waste and sample management; and establishing and strengthening COVID-19 testing quality 
assurance systems. Major impediments to this system are inadequate resources, specifically the lack of test kits and an ineffective sample transportation system to the few available testing centers.

The success of the Rwandan laboratory system has been attributed to population and governmental goodwill, research-based actions, optimized use of available human resources, and the use of limited resource funding models to support the established public laboratory and health system governance structures.[25] In this regard, there was very little available literature on how Zimbabwe approached scaling up research-based testing. The South African COVID-19 laboratory response relies on sufficient resources, now available at the national level, and includes routine genomic typing of the COVID-19 variants as part of surveillance.

Role of private sector in national response The COVID-19 response in all three countries has seen governments working closely with stakeholders from across the private sector, civil society, academia, professional associations, the private nonprofit sector, community-based organizations and international organizations. In all three countries, for example, a key role for the private for-profit healthcare systems has been in the provision of COVID-19-related treatment and care;[26] PPE for medical staff in under-resourced hospitals; and rapid test kits, hand sanitizers and food hampers to vulnerable communities.[27,28] There was no literature in our review showing any marked differences in private sector engagement in COVID-19 responses in all three countries.

Points of Entry All three countries are in compliance with WHO International Health Regulations (IHR).[29] The purpose of the IHR is to "prevent, protect against, control and provide a public health response to the international spread of disease in ways that are commensurate with and restricted to public health risks, and which avoid unnecessary interference with international traffic and trade." In Rwanda, a negative COVID-19 PCR test is required at any of the entrance points. A repeat test is conducted upon arrival while travelers are in a mandatory 24-hour quarantine.

South Africa and Zimbabwe require a negative COVID-19 PCR test taken 72 and 48 hours, respectively, prior to arrival. No retesting is conducted at the airport. The Zimbabwean point-of-entry approach leverages a mature strategic information (SI) system. (The Zimbabwean port-of-entry screening system has screened over 120,000 individuals over the 6 months from June through November, 2020). The festive season period saw the ZimbabweSouth Africa Beitbridge border post experiencing a huge influx of people crossing the border. There were reports of a high number of fake COVID-19 clearance certificates by travelers from Zimbabwe resulting in South African authorities resorting to testing every traveler passing through the border post before allowing them to enter the country.[30] Another threat faced by both Zimbabwean and South African COVID-19 responses are people entering both countries using undesignated entry points. The Rwandan strategy of repeat testing could help South Africa and Zimbabwe address the threat of the fake COVID-19 certificates.

COVID-19 logistics, supply and procurement implementation/operational plan We found very little literature on COVID-19 logistics, supply and procurement implementation in the three countries. The available evidence suggests that all three coun- tries have set up systems to map available resources and supply systems in their healthcare sectors. However, just like other African countries, Rwanda, South Africa and Zimbabwe have been affected by shortages of diagnostic kits due to disruptions in the global supply chain.[31] Furthermore, reports of COVID-19 procurement-related corruption (concerning contracts for products and services related to COVID-19) have hampered the response in South Africa[32,33] and Zimbabwe.[34,35]

The COVID-19 response has exacerbated the need for South Africa and Zimbabwe to establish measures to curb corruption within their governments. In this regard, the Rwandan model could serve as an exemplar. The Rwandan government formulates and implements anti-corruption efforts mainly via homegrown initiatives, minimizing corruption by eradicating opportunities for misconduct, focusing on governance reforms and maintaining a zero-tolerance policy towards corruption.[36] Political will, strong leadership, the active role played by the anti-corruption agency and effective governmental reforms have made Rwanda's anti-corruption activities largely successful in the context of the pandemic.[36]

\section{DISCUSSION}

COVID-19 burdens in these countries vary, with South Africa experiencing the worst epidemic of the three. The ten comparison domains discussed above influence the burden of COVID-19 in each of the countries, albeit there are concerns on the reliability of reported data due to the poor surveillance systems in place in Africa.

In general, countries with strong, coordinated government responses have experienced far less severe COVID-19 epidemics than countries with more ad hoc or laissez faire approaches. While most African countries have under-resourced health systems, many of them also have very robust public health systems, an important asset in disease mitigation and containment during a pandemic.

Our findings revealed some critical response areas where the three countries could learn from each other. For instance, Rwandan response could learn from South Africa and Zimbabwe on inter-ministerial coordination and involve more line ministries in the national coordination taskforce to improve inter-ministerial cooperation and streamline delegation of responsibility. Regarding framing and implementing policies, South Africa and Zimbabwe could learn from Rwanda to improve their speeds in implementing and establishing COVID-19 policies and making them available in the public domain. The existence of a COVID-19 national policy that covers major response areas in the public domain or on government websites is critical to guiding the response in any country.

Zimbabwe could learn from Rwanda and South Africa in devising innovative ways to improve the health worker staff complement as these are critical frontline workers in the pandemic response. Rwanda and Zimbabwe could learn from South Africa's rapid expansion of telehealth services to ensure the continuation of health services during the lockdown period. Finally, South Africa and Zimbabwe could learn from Rwanda's response to corruption, which has hampered their two countries' supply chains and logistics. In Rwanda's case, political will and strong leadership, the active role played by the anti-corruption agency, and effective governance reform have prevented mismanagement of COVID-19 resources or procurement processes. 
Between 2015 and 2017, the doctor-inhabitant ratio improved in Rwanda, from $1: 15,428$ to $1: 8,592$, while the nurse-inhabitant ratio improved from 1:1200 to 1:1070.[37] Rwanda is among the few countries in Africa to have achieved universal health coverage based on a vision of inclusiveness, equity, and comprehensive and integrated services, with a focus on primary health care (PHC). [37] Not surprisingly, Rwanda has been ranked first in Africa and sixth globally in managing the COVID-19 pandemic and making information about the pandemic accessible to the public.[38]

According to the World Bank, Rwanda has 0.1, South Africa 0.9 , and Zimbabwe 0.2 physicians per 1000 population.[39] The same source reports that Rwanda has 1.2, South Africa 1.3, and Zimbabwe 1.9 nurses or midwives per 1000 population.[38] Regarding COVID-19 deaths per 1 million population, Rwanda has 14 (ranked 34th in Africa), South Africa 730 (ranked 1st in Africa) and Zimbabwe 77 (ranked 13th in Africa).[40] Interestingly, South Africa, with the highest proportion of physicians, also shows the highest proportion of Coronavirus deaths per 1 million. It is also worth noting that South Africa has become the first country in Africa to receive a shipment of COVID-19 vaccines.

\section{CONCLUSIONS}

Frequently, analyses about Africa are based on viewpoints formulated outside the continent. We have intentionally avoided this approach. Our perspective is based on a narrative literature review, consisting mainly of documents elaborated by African policymakers. Nevertheless, it has some limitations. Firstly, as an analysis based on a literature review, steps in systematic evidence synthesis were omitted. These include quality assessment of the findings. Second, our search was limited in scope and depth. For instance, we did not screen references in the reviewed papers. However, findings in the present study still offer important insights as to similarities and differences in COVID-19 response strategies across three countries in Africa that have experienced varying impacts from the pandemic.

The findings allow for each country's COVID-19 response leaders to learn from the others and may also serve as a guide for similar settings with limited resources on the best practices for curbing the pandemic's spread.

For example, Rwanda could learn from South Africa on strategies to ensure continuation of essential services during lockdown. South Africa and Zimbabwe could learn from Rwanda's response to corruption, a factor that has hampered the two countries' supply chains and logistics. Zimbabwe could learn from Rwanda and South Africa in devising innovative ways for strategic health worker deployment. All three countries can benefit from exchanging lessons they have been learning during the pandemic and by establishing routine meetings to share their experiences. $-1 /$

\section{REFERENCES}

1. World Health Organization [Internet]. Geneva: World Health Organization; c2021. Diseases. Coronavirus disease (COVID-19) pandemic; 2020 [cited 2020 Nov 25]. Available at: https://www.who .int/emergencies/diseases/novel-coronavirus-2019

2. Musa HH, Musa TH, Musa IH, Musa IH, Ranciaro A, Campbell MC. Addressing Africa's pandemic puzzle: perspectives on COVID-19 infection and mortality in sub-Saharan Africa. Int $\mathrm{J}$ Infect Dis. 2020 Jan;102:483-8.

3. Zimbabwe COVID-19 Operational Plan. Harare: Ministry of Health and Child Care (ZW); 2020

4. Wells C, Yamin D, Ndeffo-Mbah ML, Wenzel N, Gaffney SG, Townsend JP, et al. Harnessing case isolation and ring vaccination to control Ebola. PLoS Negl Trop Dis. 2015 May 29;9(5):e0003794. DOI: 10.1371/journal .pntd.0003794.

5. World Health Organization. Regional Office for Africa [Internet]. Geneva: World Health Organization; c2021. Health topics. Coronavirus (COVID-19); [cited 2020 Nov 25]. Available at: https://www.afro .who.int/health-topics/coronavirus-covid-19

6. World Health Organization Regional Office for Africa [Internet]. Geneva: World Health Organization; c2021. Publications. Monitoring and evaluation framework for the COVID-19 response in the WHO African Region 2020; 2020 Aug [cited 2020 Nov 23]. 24 p. Available at: https://www.afro.who int/publications/monitoring-and-evaluation-frame work-covid-19-response-who-african-region

7. Our World in Data [Internet]. Oxford: University of Oxford; c2021 [cited 2021 Feb 26]. Available at: https://ourworldindata.org/

8. Worldometer [Internet]. [place unknown]: Worldometer; c2021. Coronavirus. COVID-19 CORONAVIRUS PANDEMIC. Coronavirus cases; [updated 2020 Jan 29; cited 2020 Jan 29]. Available at: https://www.worldometers.info/coronavirus/

9. Our World in Data [Internet]. Oxford: University of Oxford. Coronavirus (COVID-19) Vaccinations; [cited 2021 Feb 26]. Available at: https://ourworld
indata.org/covid-vaccinations?country=OWID _WRL

10. Bridget $\mathrm{H}$, Julien $\mathrm{N}$, Christian $\mathrm{N}$, Pacifique $\mathrm{N}$ Nadia $\mathrm{H}$, Fidele $\mathrm{B}$, et al. COVID-19 Rwanda response updates March 14-April 25, 2020. Rw Public Health Bull. 2020 Mar;2(1):11-2.

11. Campbell J. President Ramaphosa leads strong response to COVID-19 in South Africa 2020 [Internet]. New York: Council of Foreign Relations; 2020 Apr 7 [cited 2020 Nov 25]. Available at: https://www .cfr.org/blog/president-ramaphosa-leads-strong -response-covid-19-south-africa

12. Ndoro T. Mnangagwa sets up Covid-19 Taskforce to deal with Coronavirus pandemic [Internet] Harare: Harare.com; [updated 2021 May 24]; [cited 2020 Nov 25]. Available at: https://iharare .com/mnangagwa-sets-up-covid-19-taskforce-to -deal-with-coronavirus-pandemic/

13. Bridget $\mathrm{H}$, Julien $\mathrm{N}$, Christian $\mathrm{N}$, Pacifique $\mathrm{N}$, Nadia H, Fidele B, et al. COVID-19 preparedness activities in Rwanda. Rw Public Health Bull. 2020 Mar;2(1):7-10.

14. Habinshuti $M$, Butera $Y$, Nyamwasa $D$, Musanabaganwa C, Ndishimye P, Hitimana N, et al. COVID-19 Rwanda response updates. Rw Public Health Bull. 2020 Jun;2(2):18-23.

15. South Africa's policy response to the COVID-19 pandemic [Internet]. Stellenbosch: Tralac; 2020 Jul 3 [cited 2020 Nov 25]. Available at: https:// www.tralac.org/news/article/14617-south-africa -s-policy-response-to-the-covid-19-pandemic. htmIDzobo M, Chitungo I, Dzinamarira T. COVID-19: a perspective for lifting lockdown in Zimbabwe. Pan Afr Med J. 2020 Apr;35(Suppl 2):13.

16. Truscott R. Covid-19: Health worker strikes, limited testing, and clinic closures hamper Zimbabwe's response. BMJ. 2020 Aug 19;370:m3267.

17. National Institute for Communicable Diseases [Internet]. Johannesburg: National Institute for Communicable Diseases; c2020. Symptoms monitoring and management of essential workers for COVID-19 related infection; [cited 2020
Nov 25]. Available at: https://www.nicd.ac.za/dis eases-a-z-index/covid-19/covid-19-guidelines/ symptoms-monitoring-and-management-of -essential-workers-for-covid-19-related-infection/

18. Tasamba J. Rwandan youth volunteers helping fight against COVID-19 2020 [Internet]. Ankara: Anadolu Agency; 2020 Jun 6 [cited 2020 Nov 25]; [about 2 p.]. Available at: https://www.aa.com .tr/en/africa/rwandan-youth-volunteers-helping -fight-against-covid-19/1867456

19. Pierre G, Uwineza A, Dzinamarira T. Attendance to HIV antiretroviral collection clinic appointments during COVID-19 lockdown. A single center study in Kigali, Rwanda. AIDS Behav. 2020 Dec;24(12):3299-301.

20. MoHCC. Zimbabwe MOHCC rapid assessment for COVID-19 impact on HIV service provision. Harare: Ministry of Health and Child Care, Zimbabwe; 2020.

21. Mashego P. Telehealth could be the new normal after Covid-19 2020 [Internet]. Capetown fin24; 2020 Aug 1 [cited 2021 Feb 28]; [about 2 p.]. Available at: https://www.news24.com/fin24/ companies/telehealth-could-be-the-new-normal -after-covid-19-20200801

22. Mutesa L, Ndishimye P, Butera Y, Souopgui J, Uwineza A, Rutayisire R, et al. A pooled testing strategy for identifying SARS-CoV-2 at low prevalence. Nature. 2021 Jan;589(7841):276-80.

23. Louis $E F$, Ingabire $W$, Isano $S$, Eugene $D$, Blanc J. Rwanda's response during COVID-19. Psychol Trauma Theory Res Pract Policy. 2020;5(12):565-6.

24. Ivan E, Iradukunda $P G$, Gashema $P$, Angelique I, Kabanda A, Mukantwari E, et al. Scaling up laboratory testing capacity in the context of managing emerging pandemic: lessons learned from scaling up SARS-COV-2 testing in Rwanda. Int J Innovative Sci Res Technol. 2021 Feb;6(2):339 46.

25. US News [Internet]. Washington, D.C.: US News; c2021. News World News. South Africa Govern- 
ment, Private Hospitals Agree Deal on COVID-19 Patients 2020; 2020 Jun 7 [cited 2020 Nov 25]. Available at: https://www.usnews.com/news/ world/articles/2020-06-07/south-africa-govern ment-private-hospitals-agree-deal-on-covid -19-patients

26. Africa Petrochemicals [Internet]. Pretoria: Africa Petrochemicals; c2021. Latest news. SA private sector's exemplary COVID-19 response 2020; 2020 May 13 [cited 2020 Nov 25]; [about 2 p.]. Available at: https://africanpetrochemicals .co.za/sa-private-sectors-exemplary-covid-19-res ponse/

27. Corporate Council on Africa [Internet]. Washington, D.C.: Corporate Council on Africa; c2021. News. South African government and private sector response to COVID-19 with H.E. Nomaindiya Mfeketo, Ambassador of South Africa to the U.S.; 2020 Jun 11 [cited 2020 Nov 23]. Available at: https://www.corporatecouncilonafrica .com/news/south-african-government-and-pri vate-sector-response-covid-19-he-nomaindiya -mfeketo-ambassador

28. World Health Organization [Internet]. Geneva: World Health Organization; c2021. Publications. Overview. International Health Regulations (2005) Third Edition; 2016 Jan 1 [cited 2020 Nov 23]. 91 p. Available at: https://www.who.int/ publications/i/item/9789241580496

29. Mphisa R. SA rejecting Zimbabwe COVID-19 test certificates [Internet]. Harare: NewsDay; 2021 Jan 7 [cited 2021 Jan 7]. Available at: https:// www.newsday.co.zw/2021/01/sa-rejecting-zim babwe-covid-19-test-certificates/

30. Dzinamarira T, Mukwenha S, Eghtessadi R, Cuadros DF, Mhlanga G, Musuka G. Coronavirus disease 2019 [COVID-19] response in Zimbabwe: a call for urgent scale-up of testing to meet national capacity. Clin Infect Dis. 2021 May 18:72(10):e667-e74.

31. Williams-Elegbe S. Corruption, procurement and COVID-19 in Africa 2020 [Internet]. Open Ownership; 2020 Jun [cited 2020 Nov 23]; [about 3 p.]. Available at: https://www.openownership.org/ blogs/corruption-procurement-and-covid-19-in -africa/

32. Medical Brief [Internet]. Greendale (ZA): Medical Brief; c2021. South Africa. AG reveals scale of COVID-19 procurement corruption;
UIF execs suspended; 2020 Sep 2 [cited 2020 Nov 23]. Available at: https://www.medicalbrief .co.za/archives/ag-reveals-scale-of-covid-19-pro curement-corruption-uif-execs-sacked/

33. Gagné-Acoulon S. Zimbabwe's health minister charged for Covid-19 corruption 2020 [Internet]. Sarajevo: Organized Crime and Corruption Reporting Project; 2020 Jun 23 [cited 2020 Nov 23]. Available at: https://www.occrp.org/ en/daily/12594-zimbabwe-s-health-minister -charged-for-covid-19-corruption

34. Zimbabwean president fires health minister over corruption scandal 2020. Beijing: XinhuaNET; 2020 Jul 8 [cited 2020 Nov 23]. Available at: http://www .xinhuanet.com/english/2020-07/08/c_139195633 .htm

35. Oyamada E. Combating corruption in Rwanda: lessons for policy makers. Asian Educ Develop Studies. 2017 Jul;6(7). DOI: 10.1108/AEDS-03 -2017-0028

36. Alliance for Health Policy and Systems Research; World Health Organization. PRIMARY HEALTH CARE SYSTEMS (PRIMASYS). A case study from Rwanda. Geneva: World Health Organization; 2017 [cited 2020 Nov 23]. 11 p. Available at: https://www.who.int/alliance-hpsr/projects/AHP SR-PRIMASYS-Rwanda-Abridged.pdf?ua=1

37. Africanews [Internet]. Lyon: Africanews; c2021. News. Rwanda ranked first in Africa, sixth globally in Covid-19 management; [updated 2021 Jan 29]; [cited 2020 Feb 28]. Available at: https://www.africanews.com/2021/01/29/rwan da-ranked-first-in-africa-sixth-globally-in-covid -19-management/?s=09

38. The World Bank [Internet]. New York: The World Bank; c2021. Data. Physicians per 1000 population; [cited 2020 Feb 28]. Available at: https:// data.worldbank.org/indicator/SH.MED.PHYS .ZS?locations

39. Worldometer [Internet]. [place unknown]: Worldometer; c2021. Coronavirus. COVID-19 CORONAVIRUS PANDEMIC. Coronavirus cases; [updated 2020 Jan 29; cited 2020 Feb 29]. Available at: https://www.worldometers.info/coronavirus/

\section{THE AUTHORS}

Tafadzwa Dzinamarira, public health expert with a master's degree in public health and a doctorate in health sciences. Technical Advisor at ICAP @ Columbia University, Rwanda and an Extraordinary Lecturer in the School of Health Systems and Public Health, University of Pretoria, South Africa. https://orcid.org/0000-0002 $-9929-5739$

Munyaradzi P. Mapingure, public health expert with a master of science in medicine degree. Project Director, Columbia University ICAP Program, Zimbabwe. https://orcid.org/0000-0002 $-7777-9492$

Gallican N. Rwibasira, physician with a master's in public health. Division Manager of HIVI AIDS and STI Diseases at the Rwanda Biomedical Center, Rwanda. https://orcid.org/0000 $-0002-1559-6527$

Solomon Mukwenha, public health laboratory expert with a master's degree in public health. Technical Advisor, Columbia University ICAP Program, Zimbabwe. https://orcid.org/0000 $-0001-6260-8247$

Godfrey Musuka (Corresponding author: gm2660@cumc.columbia.edu), Doctor of Veterinary Medicine, Master of Philosophy and a master of science in medicine. Country Director Columbia University ICAP Program, Zimbabwe. https://orcid.org/0000-0001-9077-4429

Submitted: January 12, 2021

Approved for publication: June 22, 2021 Disclosures: None 